\title{
Prioritising Risk Factors for Type 2 Diabetes: Causal Inference through Genetic Approaches
}

\author{
Laura B. L. Wittemans ${ }^{1} \cdot$ Luca A. Lotta $^{1} \cdot$ Claudia Langenberg $^{1}$ \\ Published online: 19 May 2018 \\ (C) The Author(s) 2018
}

\begin{abstract}
Purpose of the Review Causality has been demonstrated for few of the many putative risk factors for type 2 diabetes (T2D) emerging from observational epidemiology. Genetic approaches are increasingly being used to infer causality, and in this review, we discuss how genetic discoveries have shaped our understanding of the causal role of factors associated with T2D.

Recent Findings Genetic discoveries have led to the identification of novel potential aetiological factors of T2D, including the protective role of peripheral fat storage capacity and specific metabolic pathways, such as the branched-chain amino acid breakdown. Consideration of specific genetic mechanisms contributing to overall lipid levels has suggested that distinct physiological processes influencing lipid levels may influence diabetes risk differentially. Genetic approaches have also been used to investigate the role of T2D and related metabolic traits as causal risk factors for other disease outcomes, such as cancer, but comprehensive studies are lacking.

Summary Genome-wide association studies of T2D and metabolic traits coupled with high-throughput molecular phenotyping and in-depth characterisation and follow-up of individual loci have provided better understanding of aetiological factors contributing to $\mathrm{T} 2 \mathrm{D}$.
\end{abstract}

Keywords Genetics $\cdot$ Causal risk factors $\cdot$ Type 2 diabetes $\cdot$ Mendelian randomisation

\section{Introduction}

\section{The Urgency to Prioritise Proposed Risk Factors}

For very few of the many type 2 diabetes (T2D) risk factors $[1-6,7 \cdot]$ that have emerged from observational research has causal relevance been demonstrated [8-12]. Randomised controlled trials are the "gold-standard" for causality assessment but are expensive and time-consuming, and may not be feasible (e.g. birth weight, epigenetic markers) or ethical (e.g.

This article is part of the Topical Collection on Genetics

Claudia Langenberg

Claudia.Langenberg@mrc-epid.cam.ac.uk

Laura B. L. Wittemans

Laura.Wittemans@mrc-epid.cam.ac.uk

Luca A. Lotta

Luca.Lotta@mrc-epid.cam.ac.uk

1 MRC Epidemiology Unit, University of Cambridge School of Clinical Medicine, Box 285 Institute of Metabolic Science, Cambridge Biomedical Campus, Cambridge CB2 0QQ, UK alcohol consumption, smoking) for some exposures. Examples of high-profile failures to replicate interventions that were prioritised on the basis of observational evidence highlight the limited ability of even rigorously conducted observational epidemiological studies to control the influence of bias and confounding and allow inference about causality of the observed statistical associations [13, 14]. While risk factors that are not causally associated with a disease, for example markers of the subclinical disease process, can be useful for disease prediction, this is not the case if the aim is to identify targets for intervention. Hence, new ways to prioritise risk factors on the basis of their causal likelihood are needed.

\section{Genetic Approaches to Assess Causality}

Genetic evidence through genotyping and sequencing of patients is expected to reduce the high rate of late-stage failures in drug development to translate laboratory models into the clinic [15-17]. Similarly, genetic insights may help to generate evidence about causal relevance and prioritise risk factors for testing in trials. Genetic approaches can support causal inference because genetic variants are generally less correlated 
with many of the measured and unmeasured factors that can confound observational exposure-to-disease associations, provided important assumptions are met (Fig. 1). This genetic approach to causal inference, referred to as "Mendelian randomisation", was originally proposed for the investigation of the association between fibrinogen and coronary heart disease by Keavney [18] and has since gained popularity and been the subject of many investigations and reviews [19-22]. Genetic approaches to estimate causal effect sizes require estimates of genetic associations with exposures of interest and outcomes. For many risk factors, these data have already been generated through genome-wide association studies (GWAS), and results for T2D are publically available via the online type 2 diabetes knowledge portal (http://www. type2diabetesgenetics.org) [23]. Therefore, the cost and time investment is relatively small, specifically considering the costs, duration and participant burden of trials.

GWAS of T2D have clearly demonstrated the importance of established aetiological pathways, specifically pancreatic beta cell function, adiposity and insulin resistance [24••, 25, 26•]. This suggests that hypothesis-free genetic approaches provide a useful tool to identify and assess causality of novel disease mechanisms.

\section{Outline}

This review focuses on aetiological pathways of T2D emerging from large-scale GWAS and richly phenotyped epidemiological studies, using three illustrative examples. We outline how recent genetic research has revealed a complex role of lipid metabolism in the aetiology of diabetes and has proposed peripheral fat storage capacity as a protective factor for T2D. We then describe how genetics can help to identify and prioritise causal pathways among the multitude of established and emerging biomarkers. Finally, we highlight the opportunities to use insights into the genomics of diabetes to infer its relevance as a causal risk factor for other common serious conditions, such as cancer.

\section{The Central Role of Peripheral Fat}

\section{Overall Adiposity and Fat Distribution Both Affect Diabetes Risk}

Obesity is a strong modifiable risk factor for T2D [8-12] and responsible for the secular increase in the diabetes prevalence, with three quarters of people with diabetes now living in lowand middle-income countries [27]. Weight loss interventions reduce diabetes risk [8-12] and are even able to revert glycaemia in patients with T2D to non-diabetic levels [28]. Genetic evidence supports the causal role of overall adiposity; the major body mass index (BMI) locus near FTO was the first found to exert its diabetes risk-raising effect entirely by increasing BMI. More recently, the largest European ancestryfocused GWAS of T2D, which tested 27 million variants and included more than 74,000 cases showed that 26 of the more than 400 conditionally independent variants at the identified loci for T2D predominantly increase risk through their effect on BMI, including established adiposity loci such as FTO, $M C 4 R$ and TMEM18 [24••]. Independent causal roles of overall adiposity and abdominal fat accumulation has also been supported by Mendelian randomisation studies [29•, 30, 31•].

\section{Genetic Loci Link Low Adiposity to High Diabetes Risk}

Evidence is emerging that not all mechanisms leading to reduced fat accumulation are uniformly beneficial to metabolic health. Loci near IRS1 and COBLL1/GRB14 identified for lower total body fat percentage were found to be associated with higher risk of T2D $[32 \bullet, 33]$. Similarly, a coding variant in the CREBRF gene was found to increase BMI by 1.32 $1.46 \mathrm{~kg} / \mathrm{m}^{2}$ per allele but reduce diabetes risk by $40 \%$ in a Polynesian population [34•]. In line with this, the most recent T2D GWAS showed that associations for a subset of loci were strengthened following adjustment for body mass index; among these were signals previously shown to be associated with lower capacity of adipose tissue generation and expansion [35••], including PPARG - a gene involved in Mendelian forms of partial lipodystrophy [36]. Together, these results suggest that, when focussing on distinct pathways connecting adiposity and diabetes, certain biological processes may reduce fat accumulation while increasing risk of T2D, while others will reduce risk.

\section{Lipodystrophy-Like Mechanisms May Contribute to Insulin Resistance}

Genetic research on metabolic risk factors demonstrated that distinct pathways linking low adiposity to diabetes risk may be driven by insulin resistance. It was recognised early on that discovery of loci associated with insulin resistance was facilitated by accounting for differences in obesity levels [37]; GWAS of insulin resistance have since been conducted based on fasting insulin levels adjusted for BMI [35••, 37-39]. Research on the relationship between insulin resistance and refined measures of body composition measured by dual energy X-ray absorptiometry (DEXA) showed associations between genetic determinants of insulin resistance and reduced fat accumulation in the legs and other peripheral compartments [35••, 39]. Positive relationships of insulin resistance with visceral fat mass and liver enzymes [35••, 39, 40] suggested that genetic predisposition to insulin resistance, not primarily driven by a long-term positive energy balance, is linked to a body fat distribution pattern that favours visceral and ectopic fat deposition over deposition in peripheral body 


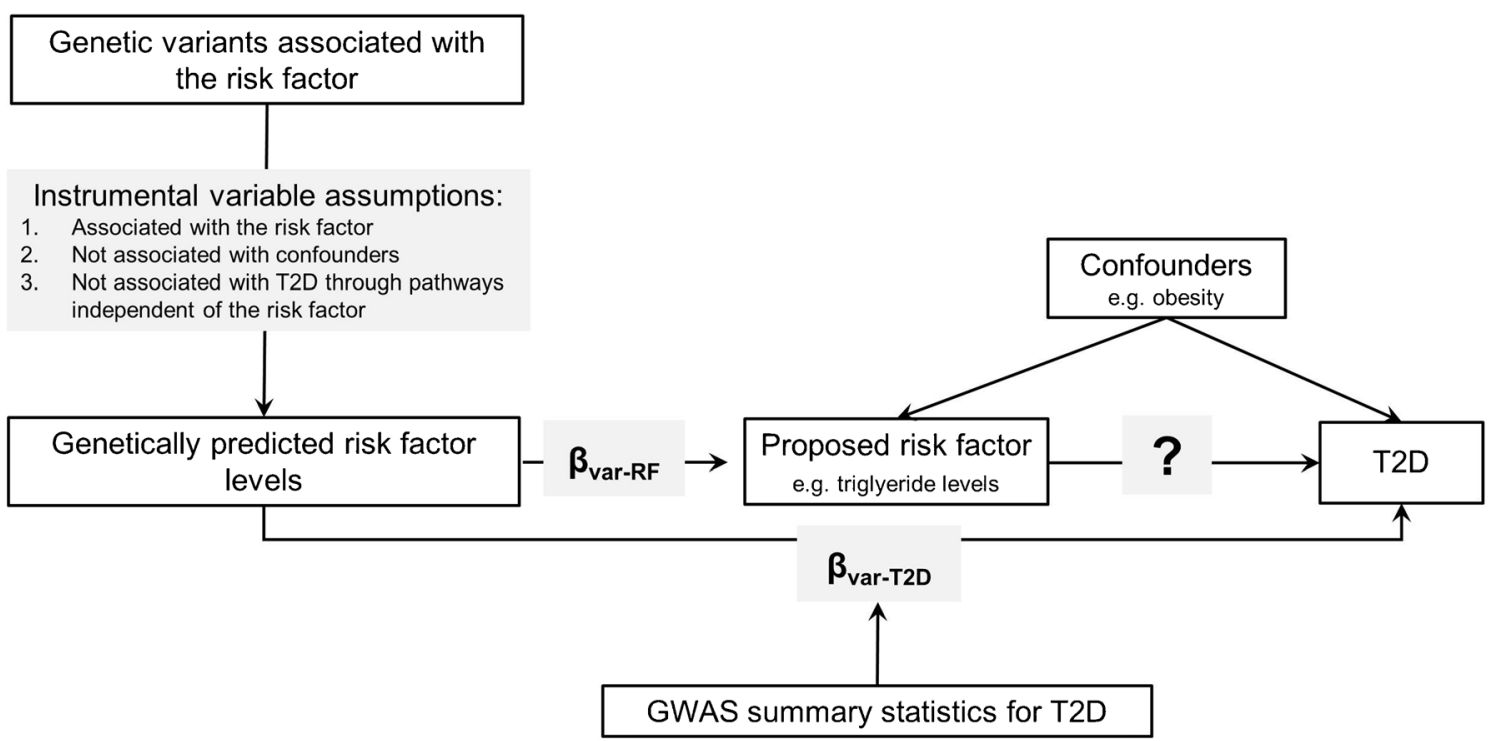

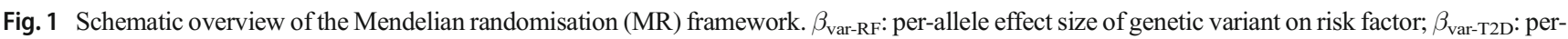
allele effect size of genetic variant on T2D

compartments. Questions remain on whether impaired peripheral fat and enhanced central fat distribution are independent mechanisms.

Lotta et al. found that the 53 loci associated with hallmarks of common insulin resistance are also enriched in patients with familial partial lipodystrophy type 1, which is a rare condition characterised by extreme insulin resistance and the limited ability to store fat peripherally [35••]. These loci identified for common insulin resistance were enriched with causal genes involved in monogenic forms of lipodystrophy, including PPARG, PIK3R1 and INSR [41]. This suggests the presence of shared mechanisms between common and rare forms of insulin resistance, and that lipodystrophy-like mechanisms may contribute to common forms of insulin resistance and metabolic and cardiovascular disease.

The majority of genetic studies have been conducted in participants of European ancestry. Many people who develop T2D in South-East Asia, the middle East and sub-Saharan Africa are not overweight or obese [42-44]. Ethnicity-specific patterns of fat distribution may underlie differences in metabolic risk for a given BMI, but the causal role of the relative contributions of greater abdominal and lack of gluteo-femoral fat have not been investigated.

\section{The Adipose Tissue Expandability Hypothesis}

Altogether, these findings support the so-called adipose tissue expandability hypothesis, which states that each individual has a limited capacity to store excess energy in adipose tissue; once that threshold has been reached, excess energy in the form of lipids may accumulate ectopically in diabetes-relevant tissues such as the liver, muscles and the pancreas, where they may contribute to tissue dysfunction and diminished insulin action
$[45,46]$. Individuals with a larger capacity to peripherally accumulate fat may therefore be more protected from the cardiometabolic consequences of a long-term positive energy balance. In support of this hypothesis, longitudinal data on weight change have demonstrated that people with a higher burden of insulin resistance-increasing alleles tend to expand their hip fat depot less as they gain weight than people at low genetic risk to develop insulin resistance [35••]. Refined imaging methods, including DEXA and magnetic resonance imaging (MRI), will help to further confirm this hypothesis and the specific contribution of these mechanisms to the broader relationship between fat distribution and metabolic risk in men and women and across ethnic groups.

\section{The Complex Interplay between Blood Lipid Fractions and T2D}

\section{Genetics-Based Causality Assessments of Blood Lipids as Diabetes Risk Factors}

After meta-analyses of randomised controlled trials of statins revealed that this major class of low-density lipoprotein cholesterol-lowering drugs modestly increases risk of T2D [47], a series of genetic studies focussing on the contribution of lipid fractions to diabetes risk was conducted. Early genetic studies investigating the causality of the blood lipid fractions focussed on the individual, separate contributions of three major lipid fractions: total triglycerides, high-density lipoprotein (HDL) and low-density lipoprotein (LDL) cholesterol [48-50]. Because levels of these fractions are regulated by partially overlapping physiological processes, genetic scores were not specific to one lipid fraction only, and therefore, 
specific causal inference is limited. To disentangle the causal effect of genetically related risk factors, the so-called multivariable Mendelian randomisation methods have been developed [51•], which estimate independent effect sizes of genetically correlated risk factors on the outcome in one model by taking the genetic correlation between them into account. High HDL cholesterol has been consistently associated with lower incidence of T2D, both observationally [52-54] and in multivariable Mendelian randomisation studies [55••, 56••], but recent evidence highlights that the extent to which LDL cholesterol and total triglyceride levels influence diabetes risk may be mechanism-dependent.

\section{Complex Relationships between LDL Cholesterol and Diabetes Risk}

While there is no doubt that higher LDL cholesterol increases the risk of diseases of the heart and vasculature, strong evidence is now available that some mechanisms lowering LDL

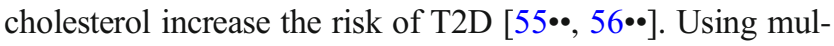
tivariable Mendelian randomisation, two recent publications suggested that higher levels of LDL cholesterol are causally related to lower diabetes risk, in line with evidence of statin trials, [47] and a study of patients with familial hypercholesterolaemia, reporting a lower incidence of $\mathrm{T} 2 \mathrm{D}$, compared to their unaffected family members [57]. In contrast, familial hypercholesterolaemia was not associated with lower diabetes risk in an Amish population [58]. Differences in the genetic background, identification and management of these Dutch and Amish patient populations may have contributed to these discrepant findings.

The extent to which levels of LDL cholesterol influence diabetes risk may depend on the physiological mechanism that contributes to differences in lipid levels [59••, 60••]. For a given effect on LDL, the effect size of the associations between genetically predicted LDL cholesterol and T2D was shown to differ depending on the gene in which LDL variants are located when variants in current or prospective LDLlowering drug targets, including PCSK9, HMGCR, NPC1L1, $A B C G 5 / G 8$ and $L D L R$, were compared [59・•]. This is in contrast to the effect of LDL cholesterol on risk of coronary heart disease, which follows a clear log-linear pattern, in observational, genetic $[59 \bullet \bullet, 61]$ and intervention studies [62].

\section{The Role of Total Triglycerides May Be Mechanism-Dependent}

Although total triglycerides have been consistently associated with higher incidence of T2D [52-54], results for total triglycerides from genetics-based causal assessments are inconsistent $[55 \bullet \bullet, 56 \bullet$. Genetic evidence suggests that triglycerides may be protective or detrimental for diabetes risk, depending on the physiological mechanism driving triglyceride levels. Triglyceride-lowering genes involved in intravascular lipolysis, including intravascular lipoprotein lipase $(L P L)$ and its inhibitor angiopoietin-like 4 (ANGPTL4), have been associat-

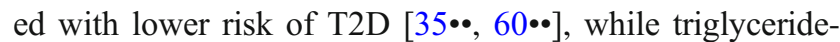
lowering variants in genes involved in the hepatic production of triglyceride-rich lipoprotein particles were linked to higher risks of diabetes and fatty liver [60••]. In contrast, there is strong genetic evidence that several triglyceride-raising mechanisms are causally related to risk of coronary heart disease $[60 \bullet \cdot, 63,64]$.

These recent findings demonstrate how different genetic approaches can help to investigate and better understand the complex relationships between dyslipidaemia and diabetes risk by looking not only at risk factor levels, but specific mechanisms underlying these differences. Investigation of combined genetic scores that encompass a large range of biological mechanisms affecting overall lipid concentrations may help general inference about causality of the exposure in question but cannot reveal insight into the complexity underlying the regulation of lipid metabolism. Given that LDL loci (for a given effect on LDL) differed in their association with diabetes risk, locus-specific results may also help to better estimate the effect of interventions that target levels through different mechanisms will have on different outcomes. Pleiotropy and heterogeneity of genetic loci is considered a limitation of Mendelian randomisation, but studies on the role of lipid metabolism for diabetes aetiology highlight that biologically relevant insights can be obtained by investigating individual genetic loci that are pleiotropic or have, given their effect on the exposure, a disproportionate effect size on the outcome.

\section{Genetics to Prioritise Causal Pathways Amongst the Multitude of Reported Biomarker Associations}

\section{Biomarkers as Causal Candidates}

Interest into the potential causality of biomarkers for T2D has grown, as they can point to novel disease pathways and help to identify potential targets for intervention, provided they are causal. A systematic literature review has shown that more than 160 biomarkers measured in blood or urine have been proposed to be associated with $\mathrm{T} 2 \mathrm{D}$, but for a small minority has an assessment of causality been attempted [7•]. No evidence for causality was found for the majority of the biomarkers that have been investigated using genetic approaches, including Vitamin D [65], adiponectin [66, 67], uric acid [68], C-reactive protein [69] and gamma-glutamyl transferase [70-72]. However, for a few biomarkers, including sex hormone-binding globulin [73, 74], B and A-type natriuretic peptide systems [75, 76] and bilirubin [77], suggestive 
evidence for a causal relationship with T2D has been reported. Historically, some of the older studies investigating one biomarker at a time were based on comparatively small sample sizes and a limited understanding of the specificity of the genetic score. Replication of these findings is needed using genetic prediction models that maximise exposure variance explained, utilise largest available GWAS summary statistics, test generalisability across different ancestries where possible and investigate specificity of genetic variants in adequately powered studies with comprehensive coverage of relevant biological pathways.

\section{New Wave of Omics-Based Biomarkers}

Technological developments in "-omics" methods have enabled high-throughput assessment of molecular traits in large epidemiological studies, including the metabolome, proteome and lipidome. This has led to a new wave and scale of observational diabetes "biomarker" discoveries, including metabolites [78-82], lipid species [83-86], proteins [87] and methylation markers [88•]. Molecular traits, such as metabolites, often represent the same biological pathway; therefore, large subsets of traits can be strongly correlated with each other. Cohort studies assessing different outcomes have shown that individual molecular traits tend to be associated with multiple disease outcomes [89-91]. The lack of specificity with regard to the exposure and outcome highlights that largescale parallel biomarker assessment in an observational setting is subject to the same limitations as traditional biomarker studies and is even more prone to falsepositive discovery by multiple testing. Integration of genetic data can be a helpful tool to prioritise potential causal pathways from the dense network of molecular trait-todisease outcome associations.

\section{Omics Integration}

GWAS have been conducted on the metabolome [92-94], proteome [95••] and methylome [96], with larger scale efforts underway. Results of these studies show that, due to the close biological proximity, genetic loci explain large proportions of the variance of a given marker and relevant biological mechanisms can be identified in relatively small sample sizes. For example, in a recent study on the genetic architecture of the human plasma proteome, GWAS were conducted for nearly 3000 proteins, based on 3000 participants [95••]. Despite the relatively modest sample size, more than 1900 genetic associations with 1478 proteins were found, and $66 \%$ of the identified regions influenced protein levels encoded by nearby genes. The median variance in protein levels explained by the variants was $5.8 \%$ (interquartile range $2.6-12.4 \%$ ), which is similar the total variance in BMI explained by over 550 signals identified in a GWAS of around 700,000 participants [97].

Integration of genetic markers with large-scale molecular phenotyping can enable the identification of novel, molecular aetiological pathways. For example, GWAS in combination with metabolomics was recently used to assess the causality of the widely reported associations between branched-chain amino acids and incidence of T2D [89]. Based on a genetic score including variants in the PPM1K gene encoding the activator for the enzyme catalysing the rate-limiting step in branched-chain amino acid breakdown, evidence was found that branched-chain amino acid catabolism and T2D may be bidirectionally causally related [98•]. In another study, evidence based on cis-acting genetic markers was found that the protein marker macrophage metalloelastase 12 (MMP-12) may protect against coronary heart disease, while observational studies suggested a positive association [95••]. This finding suggests potential safety issues for MMP-12 inhibitors, which are being developed for the treatment of chronic obstructive pulmonary disease. Finally, Elliott et al. identified genetic loci associated with methylation markers previously associated with T2D and used these as genetic instruments to distinguish methylation markers that may be on the causal path to T2D from those that are likely to be non-causal [99••]. Their results suggested that the vast majority of methylation markers for diabetes are unlikely to be causal, with the exception of a methylation site at the KCNQ1 locus [99••].

As -omics technologies are becoming more widely implemented in epidemiological studies of increasing size, opportunities to obtain an even more comprehensive insight into disease mechanisms may arise by integrating multiple layers of -omics data.

\section{The Causal Interplay between Diabetes, Metabolic Risk Factors and Cancer Incidence}

Obesity [100] and T2D [101] have been linked to higher incidence of several cancers, but the causal role of metabolic health in cancer risk is difficult to assess observationally due to bias and confounding. Ongoing efforts by the Meta-Analyses of Glucose and Insulinrelated traits Consortium (MAGIC) and others continue to improve our understanding of the genetic determinants of phenotypes associated with adiposity and T2D that are likely to contribute to the links between obesity, T2D and cancer. Application of these discoveries to results from GWAS identifying germline variants associated with site-specific cancer risk may help to refine our understanding of relevant pathways and identify 
appropriate preventative interventions. Such efforts critically depend on willingness and ability to make genome-wide summary statistics accessible for researchers in a timely manner. As mentioned above, the T2D research community systematically contributes new findings to an openly accessible online knowledge platform [23]. A similar dedicated online platform for cancer GWAS summary results and a more systematic deposition of GWAS summary results to existing online tools such as Phenoscanner (http://www.phenoscanner. medschl.cam.ac.uk/phenoscanner) [102] or MR Base (www.mrbase.org) [103] would be of great benefit to the scientific community and facilitate rapid and comprehensive investigation of the links between metabolic risk factors and cancer across different cancer sites and types.

Genetics-based research has proposed adiposity and insulin resistance as causal risk factors for specific types of cancer. Adiposity, insulin resistance and secretion have all been shown to be causally related to endometrial cancer risk, the site most strongly associated with obesity observationally $[104,105]$. For breast cancer, a protective effect of body mass index in both pre- and post-menopausal breast cancer has been reported [106], but no causal role for T2D was found $[107,108]$. Genetically predicted insulin resistance has also been associated with lung [109•] and pancreatic cancer [110], whereas genetically predicted BMI has been linked to higher risk of colorectal [111•], pancreatic [110] and lung cancer [109•].

These early genetics-based studies suggest that distinct metabolic risk factors may be causal for different types of cancer, but comprehensive investigations that assess the role of a range of refined metabolic risk factors across cancer types are still lacking.

\section{Conclusion}

Rapid progress in the identification of the genetic basis of T2D and related phenotypes, together with technological advances that facilitate high-throughput and refined phenotyping at scale, provide important opportunities for epidemiological research to identify novel aetiological pathways and enhance causal understanding of potential risk factors that are amenable to intervention. Better powered studies now provide greater sensitivity to investigate pleiotropy that may have previously been overlooked and may have confounded effect sizes of genetically predicted exposures. Traditional genetic approaches to infer causality have generally focused on average effects, sometimes estimated from large polygenic scores. Recent examples have highlighted opportunities that arise from distinguishing the effects of specific mechanisms represented by a locus or a subset of loci, which may otherwise be overlooked.

\section{Compliance with Ethical Standards}

Conflict of Interest Laura B.L. Wittemans, Luca A. Lotta and Claudia Langenberg declare that they have no conflict of interest.

Human and Animal Rights and Informed Consent This article does not contain any studies with human or animal subjects performed by any of the authors.

Open Access This article is distributed under the terms of the Creative Commons Attribution 4.0 International License (http:// creativecommons.org/licenses/by/4.0/), which permits unrestricted use, distribution, and reproduction in any medium, provided you give appropriate credit to the original author(s) and the source, provide a link to the Creative Commons license, and indicate if changes were made.

\section{References}

Papers of particular interest, published recently, have been highlighted as:

- Of importance

- Of major importance

1. Willi C, Bodenmann P, Ghali WA, Faris PD, Cornuz J. Active smoking and the risk of type 2 diabetes - a systematic review and meta-analysis. JAMA. 2007;298:2654-64.

2. Hu FB, Manson JE, Stampfer MJ, Graham C, Liu S, Solomon CG, et al. Diet, lifestyle, and the risk of type 2 diabetes mellitus in women. N Engl J Med. 2001;345:790-7.

3. Hu FB, Li TY, Colditz GA, Willett WC, Manson JE. Television watching and other sedentary behaviors in relation to risk of obesity and type 2 diabetes mellitus in women. J Am Med Assoc. 2003;289:1785-91.

4. The InterAct Consortium. Dietary fibre and incidence of type 2 diabetes in eight European countries: the EPIC-InterAct Study and a meta-analysis of prospective studies. Diabetologia. 2015;58: $1394-408$.

5. O'Connor L, Imamura F, Lentjes MAH, Khaw K-T, Wareham NJ, Forouhi NG. Prospective associations and population impact of sweet beverage intake and type 2 diabetes, and effects of substitutions with alternative beverages. Diabetologia. 2015;58:147483.

6. Emdin CA, Anderson SG, Woodward M, Rahimi K. Usual blood pressure and risk of new-onset diabetes evidence from 4.1 million adults and a meta-analysis of prospective studies. J Am Coll Cardiol. 2015;66:1552-62.

7. Abbasi A, Sahlqvist A-S, Lotta L, et al. Aetiological and predictive biomarkers of the risk of developing type 2 diabetes: a systematic review. PLoS One. 2016;11:e0163721. A systematic literature review of blood-based and urinary biomarkers reported for type 2 diabetes, and an evaluation of the evidence for predictive value and causality for each of the reported biomarkers.

8. Lindström J, Louheranta A, Mannelin M, Rastas M, Salminen V, Eriksson J, et al. The Finnish Diabetes Prevention Study (DPS). Diabetes Care. 2003;26:3230-6. 
9. Diabetes Prevention Program Research Group. Reduction in the incidence of type 2 diabetes with lifestyle intervention or metformin. N Engl J Med. 2002;346:393-403.

10. Ramachandran A, Snehalatha C, Mary S, Mukesh B, Bhaskar AD, Vijay V, et al. The Indian Diabetes Prevention Programme shows that lifestyle modification and metformin prevent type 2 diabetes in Asian Indian subjects with impaired glucose tolerance (IDPP1). Diabetologia. 2006;49:289-97.

11. Dunbar JA, Hernan AL, Janus ED, et al. Challenges of diabetes prevention in the real world: results and lessons from the Melbourne Diabetes Prevention Study. BMJ Open Diabetes Res Care. 2015;3:e00131.

12. Li G, Zhang P, Wang J, et al. The long-term effect of lifestyle interventions to prevent diabetes in the China Da Qing Diabetes Prevention Study: a 20-year follow-up study. Lancet. 2008;371: 1783-9.

13. Hulley S, Grady D, Bush T, Furberg C, Herrington D, Riggs B, et al. Randomized trial of estrogen plus progestin for secondary prevention of coronary heart disease in postmenopausal women. JAMA. 1998;280:605-13.

14. Stampfer MJ, Colditz GA. Estrogen replacement therapy and coronary heart disease: a quantitative assessment of the epidemiologic evidence. Prev Med. 1991;20:47-63.

15. Nelson MR, Tipney H, Painter JL, et al. The support of human genetic evidence for approved drug indications. Nat Genet. 2015;47:856-60.

16. Hurle MR, Nelson MR, Agarwal P, Cardon LR. Trial watch: impact of genetically supported target selection on R\&D productivity. Nat Rev Drug Discov. 2016;15:596-7.

17. Altshuler D. Developing medicines to prevent the development and alter the course severe genetic diseases. In: Chief Medical Officer Annual Report 2016 Generation Genome. (Chapter 4); 2017.

18. Keavney B. Genetic epidemiological studies of coronary heart disease. Int J Epidemiol. 2002;31:730-6.

19. Ebrahim S, Davey SG. Mendelian randomization: can genetic epidemiology help redress the failures of observational epidemiology? Hum Genet. 2008;123:15-33.

20. Burgess S, Bowden J, Fall T, Ingelsson E, Thompson. Sensitivity analyses for robust causal inference from Mendelian randomization analyses with multiple genetic variants. Epidemiology 2017:28:30-42.

21. Bowden J, Davey Smith G, Burgess S. Mendelian randomization with invalid instruments: effect estimation and bias detection through Egger regression. Int J Epidemiol. 2015;44:512-25.

22. Bowden J, Davey Smith G, Haycock PC, Burgess S. Consistent estimation in Mendelian randomization with some invalid instruments using a weighted median estimator. Genet Epidemiol. 2016;40:304-14

23. Flannick J, Florez JC. Type 2 diabetes: genetic data sharing to advance complex disease research. Nat Rev Genet. 2016;17:535-49.

24.• Mahajan A, Taliun D, Thurner M, et al. Fine-mapping of an expanded set of type 2 diabetes loci to single-variant resolution using high-density imputation and islet-specific epigenome maps. bioRxiv 2018:245506. doi:https://doi.org/10.1101/245506. This paper describes the most recent and largest ever genomewide association study on type 2 diabetes, including more than 74,000 cases and 824,000 controls of European descent. Based on dense reference panels for imputation and integration of regulatory annotations, significant progress at fine-mapping of associated genetic loci was made.

25. Scott RA, Scott LJ, Magi R, Marullo L, Gaulton KJ, Kaakinen M, et al. An expanded genome-wide association study of type 2 diabetes in Europeans. Diabetes. 2017;66:2888-902.
26. Mahajan A, Wessel J, Willems S, et al. Refining the accuracy of validated target identification through coding variant fine-mapping in type 2 diabetes. Nat. Genet. 2018;50:559-71. In this trans-ethnic exome-wide association analysis for type 2 diabetes on 81,400 cases and 371,000 controls, 40 coding variants were identified and for 16 of these evidence based on finemapping was found that they are the causal variant at that locus.

27. International Diabetes Federation. IDF Diabetes Atlas Eighth Edition 2017.

28. Lean MEJ, Leslie WS, Barnes AC, et al. Primary care-led weight management for remission of type 2 diabetes (DiRECT): an openlabel, cluster-randomised trial. Lancet. 2017;391:541-51.

29. Dale CE, Fatemifar G, Palmer TM, et al. Causal associations of adiposity and body fat distribution with coronary heart disease, stroke subtypes, and type 2 diabetes mellitus: a Mendelian randomization analysis. Circulation. 2017;135:2373-88. Mendelian randomization study corroborating the role of both overall and central adiposity as risk factors for coronary heart disease and type 2 diabetes.

30. Corbin LJ, Richmond RC, Wade KH, Burgess S, Bowden J, Smith GD, et al. Body mass index as a modifiable risk factor for type 2 diabetes: refining and understanding causal estimates using Mendelian randomisation. Diabetes. 2016;65:3002-7.

31. Emdin CA, Khera AV, Natarajan P, Klarin D, Zekavat SM, Hsiao AJ, et al. Genetic association of waist-to-hip ratio with cardiometabolic traits, type 2 diabetes, and coronary heart disease. JAMA. 2017;317:626-34. This Mendelian randomization study found supportive evidence that waist-to-hip ratio is an independent risk factor for type $\mathbf{2}$ diabetes and coronary heart disease.

32. Lu Y, Day FR, Gustafsson S, et al. New loci for body fat percentage reveal link between adiposity and cardiometabolic disease risk. Nat Commun. 2016;7:10495. This study conducted the largest GWAS for total body fat percentage and reported for two loci opposite directions of effect on body fat percentage and diabetes risk.

33. Kilpeläinen TO, Zillikens MC, Stančákova A, et al. Genetic variation near IRS1 associates with reduced adiposity and an impaired metabolic profile. Nat Genet. 2011;43:753-60.

34. Minster RL, Hawley NL, Su CT, et al. A thrifty variant in CREBRF strongly influences body mass index in Samoans. Nat Genet. 2016;48:1049-54. In this study on a Samoan population, a coding variant in CREBRF with a very strong increasing effect on body mass index but a risk-reducing effect on type 2 diabetes was identified through GWAS and targeted sequencing.

35.• Lotta LA, Gulati P, Day FR, et al. Integrative genomic analysis implicates limited peripheral adipose storage capacity in the pathogenesis of human insulin resistance. Nat Genet. 2017;49:17-26.

Based on genetic variants identified for three hallmarks of insulin resistance, the authors find evidence that shared mechanisms may exist between common insulin resistance not primarily driven by overweight and insulin-resistant lipodystrophy syndromes.

36. Barroso I, Gurnell M, Crowley VEF, et al. Dominant negative mutations in human PPAR $\gamma$ associated with severe insulin resistance, diabetes mellitus and hypertension. Nature. 1999;402:880 3 .

37. Manning A, Hivert M-F, Scott RA, et al. A genome-wide approach accounting for body mass index identifies genetic variants influencing fasting glycemic traits and insulin resistance. Nat Genet. 2012;44:659-69.

38. Scott RA, Lagou V, Welch RP, et al. Large-scale association analyses identify new loci influencing glycemic traits and provide insight into the underlying biological pathways. Nat Genet. 2012;44:991-1005. 
39. Scott RA, Fall T, Pasko D, et al. Common genetic variants highlight the role of insulin resistance and body fat distribution in type 2 diabetes, independently of obesity. Diabetes. 2014;63:4378-87.

40. Yaghootkar H, Scott RA, White CC, et al. Genetic evidence for a normal-weight "metabolically obese" phenotype linking insulin resistance, hypertension, coronary artery disease, and type 2 diabetes. Diabetes 2014:63:4369-4377.

41. Semple RK, Savage DB, Cochran EK, Gorden P, O'Rahilly S. Genetic syndromes of severe insulin resistance. Endocr Rev. 2011;32:498-514.

42. Ramachandran A, Wan Ma RC, Snehalatha C. Diabetes in Asia. Lancet. 2010;375:408-18.

43. Babai MA, Arasteh P, Hadibarhaghtalab M, Naghizadeh MM, Salehi A, Askari A, et al. Defining a BMI cut-off point for the Iranian population: the shiraz heart study. PLoS One. 2016;11: e0160639.

44. Frank LK, Heraclides A, Danquah I, Bedu-Addo G, Mockenhaupt FP, Schulze MB. Measures of general and central obesity and risk of type 2 diabetes in a Ghanaian population. Trop Med Int Heal. 2013;18:141-51.

45. Virtue S, Vidal-Puig A. Adipose tissue expandability, lipotoxicity and the metabolic syndrome - an allostatic perspective. Biochim Biophys Acta - Mol Cell Biol Lipids. 1801;2010:338-49.

46. Danforth E. Failure of adipocyte differentiation causes type II diabetes mellitus? Nat Genet. 2000;26:13.

47. Sattar N, Preiss D, Murray HM, et al. Statins and risk of incident diabetes: a collaborative meta-analysis of randomised statin trials. Lancet. 2010;375:735-42.

48. Haase CL, Tybjærg-Hansen A, Nordestgaard BG, Frikke-Schmidt R. HDL cholesterol and risk of type 2 diabetes: a Mendelian Randomization Study. Diabetes. 2015;64:3328-33.

49. Qi Q, Liang L, Doria A, Hu FB, Qi L. Genetic predisposition to dyslipidemia and type 2 diabetes risk in two prospective cohorts. Diabetes. 2012;61:745-52.

50. Maneka N, De Silva G, Freathy RM, et al. Mendelian randomization studies do not support a role for raised circulating triglyceride levels influencing type 2 diabetes, glucose levels, or insulin resistance. Diabetes. 2011;60:1008-18.

51. Burgess S, Thompson SG. Multivariable Mendelian randomization: the use of pleiotropic genetic variants to estimate causal effects. Am J Epidemiol. 2015;181:251-60. This paper presents a multivariable Mendelian randomization method that allows to assess the independent causal role of genetically correlated exposures.

52. Mora S, Otvos JD, Rifai N, Rosenson RS, Buring JE, Ridker PM. Lipoprotein particle profiles by nuclear magnetic resonance compared with standard lipids and apolipoproteins in predicting incident cardiovascular disease in women. Circulation. 2009;119:931-9.

53. Wilson PWF, Meigs JB, Sullivan L, Fox CS, Nathan DM, D'Agostino RAB. Prediction of incident diabetes mellitus in middle-aged adults: the Framingham Offspring Study. Arch Intern Med. 2007;167:1068-74.

54. Gupta AK, Dahlof B, Dobson J, Sever PS, Wedel H, Poulter NR. Determinants of new-onset diabetes randomized in the AngloScandinavian cardiac outcomes trial- blood pressure lowering arm and the relative influence of antihypertensive medication. Diabetes Care. 2008;31:982-8.

55.• White J, Swerdlow DI, Preiss D, Fairhurst-Hunter Z, Keating BJ, Asselbergs FW, et al. Association of lipid fractions with risks for coronary artery disease and diabetes. JAMA Cardiol. 2016;1:6929. Using multivariable Mendelian randomization, the authors provide evidence that higher LDL and HDL cholesterol, and possibly also higher triglycerides, protect against type 2 diabetes.
56.• Fall T, Xie W, Poon W, et al. Using genetic variants to assess the relationship between circulating lipids and type 2 diabetes. Diabetes. 2015;64:2676-84. This earlier study also uses multivariable Mendelian randomization to assess the independent causal effect sizes of the three lipid fractions, and reported a protective effect of LDL and HDL cholesterol, but not of triglycerides, against type 2 diabetes.

57. Besseling J, Kastelein JJP, Defesche JC, Hutten BA, Hovingh GK. Association between familial hypercholesterolemia and prevalence of type 2 diabetes mellitus. JAMA. 2015;313:1029-36.

58. Xu H, Ryan KA, Jaworek TJ, et al. Familial hypercholesterolemia and type 2 diabetes in the old order Amish. Diabetes. 2017;66: 2054-8.

59.• Lotta LA, Sharp SJ, Burgess S, et al. Association between lowdensity lipoprotein cholesterol-lowering genetic variants and risk of type 2 diabetes. JAMA. 2016;316:1383-91. This study shows that LDL-lowering variants located in/near genes that represent established or emerging targets for LDL-lowering drugs increase risk of diabetes to a different extent, whereas all variants are associated with a similar risk reduction in coronary heart disease.

$60 . \bullet$ Liu DJ, Peloso GM, Yu H, et al. Exome-wide association study of plasma lipids in >300,000 individuals. Nat Genet. 2017;49:175866. In this exome-wide association analyses for lipid fractions, LDL-lowering variants were found to have a heterogeneous effect on type 2 diabetes. Associations of triglyceride-lowering variants with diabetes risk was found to be mechanismsdependent.

61. Burgess S, Freitag DF, Khan H, Gorman DN, Thompson SG. Using multivariable Mendelian randomization to disentangle the causal effects of lipid fractions. PLoS One. 2014;9:e108891.

62. Silverman MG, Ference BA, Im K, Wiviott SD, Giugliano RP, Grundy SM, et al. Association between lowering LDL-C and cardiovascular risk reduction among different therapeutic interventions. JAMA. 2016;316:1289-97.

63. Do R, Willer CJ, Schmidt EM, et al. Common variants associated with plasma triglycerides and risk for coronary artery disease. Nat Genet. 2013;45:1345-52.

64. Helgadottir A, Gretarsdottir S, Thorleifsson G, et al. Variants with large effects on blood lipids and the role of cholesterol and triglycerides in coronary disease. Nat Genet. 2016;48:634-9.

65. Ye Z, Sharp SJ, Burgess S, Scott RA, Imamura F, InterAct Consortium, et al. Association between circulating 25hydroxyvitamin D and incident type 2 diabetes: a Mendelian randomisation study. Lancet Diabetes Endocrinol. 2015;3:35-42.

66. Mente A, Meyre D, Lanktree MB, et al. Causal relationship between adiponectin and metabolic traits: a Mendelian Randomization Study in a multiethnic population. PLoS One. 2013;8:e66808.

67. Yaghootkar H, Lamina C, Scott RA, et al. Mendelian randomization studies do not support a causal role for reduced circulating adiponectin levels in insulin resistance and type 2 diabetes. Diabetes. 2013;62:3589-98.

68. Sluijs I, Holmes M V, van der Schouw YT, et al. A Mendelian Randomization Study of circulating uric acid and type 2 diabetes. Diabetes 2015:64:3028-3036.

69. Prins BP, Abbasi A, Wong A, et al. Investigating the causal relationship of C-reactive protein with 32 complex somatic and psychiatric outcomes: a large-scale cross-consortium Mendelian Randomization Study. PLoS Med. 2016;13:e1001976.

70. Noordam R, Smit RAJ, Postmus I, Trompet S, van Heemst D. Assessment of causality between serum gamma-glutamyltransferase and type 2 diabetes mellitus using publicly available data: a Mendelian randomization study. Int J Epidemiol. 2016;45:1953-60.

71. Lee YS, Cho Y, Burgess S, Davey Smith G, Relton CL, Shin S-Y, et al. Serum gamma-glutamyl transferase and risk of type 2 
diabetes in the general Korean population: a Mendelian Randomization Study. Hum Mol Genet. 2016;25:3877-86.

72. Liu J, Au Yeung SL, Lin SL, Leung GM, Schooling CM. Liver enzymes and risk of ischemic heart disease and type 2 diabetes mellitus: a Mendelian Randomization Study. Sci Rep. 2016;6: 38813.

73. Wang Q, Kangas AJ, Soininen P, et al. Sex hormone-binding globulin associations with circulating lipids and metabolites and the risk for type 2 diabetes: observational and causal effect estimates. Int J Epidemiol. 2015;44:623-37.

74. Ding EL, Song Y, Manson JE, Hunter DJ, Lee CC, Rifai N, et al. Sex hormone-binding globulin and risk of type 2 diabetes in women and men. N Engl J Med. 2009;361:1152-63.

75. Jujić A, Nilsson PM, Engström G, Hedblad B, Melander O, Magnusson M. Atrial natriuretic peptide and type 2 diabetes development-biomarker and genotype association study. PLoS One. 2014;9:e89201.

76. Pfister R, Sharp S, Luben R, et al. Mendelian Randomization Study of B-type natriuretic peptide and type 2 diabetes: evidence of causal association from population studies. PLoS Med. 2011;8: e1001112.

77. Abbasi A, Deetman PE, Corpeleijn E, et al. Bilirubin as a potential causal factor in type 2 diabetes risk: a Mendelian randomization study. Diabetes. 2015;64:1459-69.

78. Floegel A, Stefan N, Yu Z, et al. Identification of serum metabolites associated with risk of type 2 diabetes using a targeted metabolomic approach. Diabetes. 2013;62:639-48.

79. Walford GA, Ma Y, Clish C, Florez JC, Wang TJ, Gerszten RE. Metabolite profiles of diabetes incidence and intervention response in the diabetes prevention program. Diabetes. 2016;65: 1424-33.

80. Shi L, Brunius C, Lehtonen M, Auriola S, Bergdahl IA, Rolandsson O, Hanhineva K, Landberg R. Plasma metabolites associated with type 2 diabetes in a Swedish population-a case-control study nested in a prospective cohort. Diabetologia 2018 (online publication ahead of press). doi: doi:https://doi.org/ 10.1007/s00125-017-4521-y.

81. Peddinti G, Cobb J, Yengo L, Froguel P, Kravić J, Balkau B, et al. Early metabolic markers identify potential targets for the prevention of type 2 diabetes. Diabetologia. 2017;60:1740-50.

82. Lu Y, Wang Y, Ong CN, Subramaniam T, Choi HW, Yuan JM, et al. Metabolic signatures and risk of type 2 diabetes in a Chinese population: an untargeted metabolomics study using both LC-MS and GC-MS. Diabetologia. 2016;59:2349-59.

83. Forouhi NG, Koulman A, Sharp SJ, et al. Differences in the prospective association between individual plasma phospholipid saturated fatty acids and incident type 2 diabetes: the EPIC-InterAct Case-Cohort Study. Lancet Diabetes Endocrinol. 2014;2:810-8.

84. Suvitaival T, Bondia-Pons I, Yetukuri L, Pöhö P, Nolan JJ, Hyötyläinen $T$, et al. Lipidome as a predictive tool in progression to type 2 diabetes in Finnish men. Metabolism. 2018;78:1-12.

85. Rhee EP, Cheng S, Larson MG, et al. Lipid profiling identifies a triacylglycerol signature of insulin resistance and improves diabetes prediction in humans. J Clin Invest. 2011;121:1402-11.

86. Lu Y, Wang Y, Zou L, Liang X, Ong CN, Tavintharan S, et al. Serum lipids in association with type 2 diabetes risk and prevalence in a Chinese population. J Clin Endocrinol Metab. 2018;103:671-80.

87. Belongie KJ, Ferrannini E, Johnson K, Andrade-Gordon P, Hansen MK, Petrie JR. Identification of novel biomarkers to monitor $\beta$-cell function and enable early detection of type 2 diabetes risk. PLoS One. 2017;12:e182932.

88. Chambers JC, Loh M, Lehne B, Drong A, Kriebel J, Motta V, et al. Epigenome-wide association of DNA methylation markers in peripheral blood from Indian Asians and Europeans with incident type 2 diabetes: a nested case-control study. Lancet Diabetes
Endocrinol. 2015;3:526-34. This is the largest methylationwide association thus far conducted for type 2 diabetes.

89. Wang TJ, Larson MG, Vasan RS, et al. Metabolite profiles and the risk of developing diabetes. Nat Med. 2011;17:448-53.

90. Ruiz-Canela M, Toledo E, Clish CB, et al. Plasma branched-chain amino acids and incident cardiovascular disease in the PREDIMED trial. Clin Chem. 2016;62:582-92.

91. Mayers JR, Wu C, Clish CB, et al. Elevation of circulating branched-chain amino acids is an early event in human pancreatic adenocarcinoma development. Nat Med. 2014;20:1193-8.

92. Shin S-Y, Fauman EB, Petersen A-K, et al. An atlas of genetic influences on human blood metabolites. Nat Genet. 2014;46:543-50.

93. Kettunen J, Demirkan A, Würtz P, et al. Genome-wide study for circulating metabolites identifies 62 loci and reveals novel systemic effects of LPA. Nat Commun. 2016;7:11122.

94. Draisma HHM, Pool R, Kobl M, et al. Genome-wide association study identifies novel genetic variants contributing to variation in blood metabolite levels. Nat Commun. 2015;6:7208.

95.• Sun BB, Maranville JC, Peters JE, et al. Consequences of natural perturbations in the human plasma proteome. 2017 bioRxiv 134551. doi: https://doi.org/10.1101/134551. The largest GWAS thus far conducted on 3,000 plasma proteins, highlighting several examples of how integration of genetics and proteomics can increase understanding of disease mechanisms and prioritise proteins as drug targets.

96. Bonder MJ, Luijk R, Zhernakova DV, et al. Disease variants alter transcription factor levels and methylation of their binding sites. Nat Genet. 2017;49:131-8.

97. Yengo L, Sidorenko J, Kemper KE, et al. Meta-analysis of genome-wide association studies for height and body mass index in 700,000 individuals of European ancestry. bioRxiv 2018: 274654. doi:https://doi.org/10.1101/274654.

98. Lotta LA, Scott RA, Sharp SJ, et al. Genetic predisposition to an impaired metabolism of the branched-chain amino acids and risk of type 2 diabetes: a Mendelian randomisation analysis. PLOS Med. 2016;13:e1002179. This study demonstrates how integration of metabolomics and large-scale genetic discoveries for specific metabolites can propose metabolic pathways as novel disease pathways.

99.• Elliott HR, Shihab HA, Lockett GA, Holloway JW, McRae AF, Davey Smith G, et al. Role of DNA methylation in type 2 diabetes etiology: using genotype as a causal anchor. Diabetes. 2017;66: 1713-22. In this study, genetic loci associated with methylation markers previously associated with type 2 diabetes were identified and used to distinguish methylation markers that may be on the causal path to type 2 diabetes from those that are non-causal.

100. Bhaskaran K, Douglas I, Forbes H, dos-Santos-Silva I, Leon DA, Smeeth L. Body-mass index and risk of 22 specific cancers: a population-based cohort study of 5.24 million UK adults. Lancet 2014:384:755-765.

101. Pearson-Stuttard J, Zhou B, Kontis V, Bentham J, Gunter MJ, Ezzati M. Worldwide burden of cancer attributable to diabetes and high body-mass index: a comparative risk assessment. Lancet Diabetes Endocrinol. 2017;6:95-104.

102. Staley JR, Blackshaw J, Kamat MA, et al. PhenoScanner: a database of human genotype-phenotype associations. Bioinformatics. 2016;32:3207-9.

103. Hemani G, Jie Zheng J, Kaitlin H, et al. MR-Base: a platform for systematic causal inference across the phenome using billions of genetic associations. bioRxiv 2016:078972. doi: https://doi.org/ 10.1101/078972.

104. Nead KT, Sharp SJ, Thompson DJ, et al. Evidence of a causal association between insulinemia and endometrial cancer: a Mendelian randomization analysis. J Natl Cancer Inst. 2015;107: djv178. 
105. Painter JN, O'Mara TA, Marquart L, et al. Genetic risk score Mendelian randomization shows that obesity measured as body mass index, but not waist:hip ratio, is causal for endometrial cancer. Cancer Epidemiol Biomark Prev. 2016;25:1503-10.

106. Guo Y, Warren Andersen S, Shu X-O, et al. Genetically predicted body mass index and breast cancer risk: Mendelian randomization analyses of data from 145,000 women of European descent. PLoS Med. 2016;13:e1002105.

107. Zhao Z, Wen W, Michailidou K, Bolla MK, Wang Q, Zhang B, et al. Association of genetic susceptibility variants for type 2 diabetes with breast cancer risk in women of European Ancestry. Cancer Causes Control. 2016;27:679-93.

108. Hou N, Zheng Y, Gamazon ER, et al. Genetic susceptibility to type 2 diabetes and breast cancer risk in women of European and African Ancestry. Cancer Epidemiol Biomark Prev. 2012;21: $552-6$.

109. Carreras-Torres R, Johansson MBM, Haycock PC, et al. Obesity, metabolic factors and risk of different histological types of lung cancer: a Mendelian randomization study. PLoS One. 2017;12: e 0177875 . Mendelian randomization study on the role of metabolic risk factors in risk of specific types of lung cancer suggesting causal roles for body mass index in lung cancer subtypes, and for fasting insulin in overall lung cancer.

110. Carreras-Torres R, Johansson M, Gaborieau V, Haycock PC, Wade KH, Relton CL, et al. The role of obesity, type 2 diabetes, and metabolic factors in pancreatic cancer: a Mendelian Randomization Study. J Natl Cancer Inst. 2017;109:djx012. Genetic study on the causal role of metabolic risk factors in pancreatic cancer risk. Findings suggest strong effects of body mass index and fasting insulin levels.

111. Jarvis D, Mitchell JS, Law PJ, et al. Mendelian randomisation analysis strongly implicates adiposity with risk of developing colorectal cancer. Br J Cancer. 2016;115:266-72. Mendelian randomization study reporting a strong causal effect size of body mass index and waist-to-hip ratio on risk of colorectal cancer. 